\title{
QUASI-STATIC AXIAL CRUSHING BEHAVIOR OF ALUMINUM CLOSED CELL FOAM-FILLED MULTI-PACKED ALUMINUM AND COMPOSITEIALUMINUM HYBRID TUBES
}

\author{
M. Güden, H. Kavi , S. Yüksel, A. Taşdemirci \\ Department of Mechanical Engineering, \\ Izmir Institute of Technology, Gülbahçe Köyü, Urla, \\ Izmir, TURKEY 35430
}

\begin{abstract}
The axial crushing behavior of empty and Al close-cell foam-filled Al multi-tube designs (hexagonal and square) and E-glass woven fabric polyester composite and Al hybrid tubes were investigated through quasi-static compression testing. The effects of foam filling on the deformation mode and the crushing and average crushing loads of single tubes and multi-tube designs were determined. Although foam filling increased the energy absorption in single Al tube and multi-tube designs, it was not effective in increasing the specific absorbed energy over that of the empty Al tube. However, multi-tube designs were found to be energetically more effective than single tubes at similar foam filler densities, proving a higher interaction effect in multi-tube designs. Empty composite and empty hybrid tubes crushed predominantly in progressive crushing mode, without applying any triggering mechanism. Foam filling was found to be ineffective in increasing the crushing loads of the composite tubes over the sum of the crushing loads of empty composite tube and foam. However, foam filling stabilized the composite progressive crushing mode. In empty hybrid tubes, the deformation mode of the inner Al tube was found to be a more complex form of the diamond mode of deformation of empty Al tube, leading to higher crushing load values than the sum of the crushing load values of empty composite tube and empty metal tube.
\end{abstract}

\section{Introduction}

The light-weight foam filling of metallic and fiber reinforced composite columnar structures have recently taken considerable scientific interests. The foam filling of metallic thin-walled tubes increases the Specific Absorbed Energy (SAE) values over the empty tube counterparts while the efficiency of foam filling is function of the tube geometry, tube wall-foam filler bonding and foam density chosen [1-3]. The foam filling generally results in an interaction between the tube wall and foam filler and therefore increases the average crushing load of the filled-tube over the sum of the crushing loads of foam (alone) and tube (alone). The encroachment of the tube wall into the foam filler was suggested to allow an additional compression in the filler, retarding the sectional collapse of the tube [9]. Thick isotropic metal tubes generally collapse by concertina, whereas thinwalled metal tubes collapse by diamond mode of folding and the foam filling was also shown to increase the tendency for the concertina mode of folding [4-7]. Short circular composite tubes crush under compressive loads either in catastrophic (brittle) or progressive crushing mode [8]. Compression shear resulting from fiber micro-buckling and axial splitting generally leads to the catastrophic failure of composite tubes $[9,10]$. The catastrophic failure mode is characterized by an abrupt decrease in crushing loads following the initial peak-load. On the other hand, progressive crushing mode proceeds with fragmentation, splaying and folding modes [11] and results in very high levels of energy absorption associated with the large number of micro fractures that occur as the tube crushes. The crushing behavior of empty composite tubes has been widely investigated; the effects of internal and external splaying mode [12], braiding angle [13], the matrix void content [14], resin type [15] and the tube inner radius, thickness and braiding angle [16] on the SAE values were determined. Shin et. al. [17] also investigated the axial crushing and energy absorption behavior of an Al/glass fiber reinforced epoxy square composite hybrid tubes. Harte et. al. [18] investigated the compression and tensional behavior of braided glass fiber/epoxy circular tubes with and without polymer foam fillers and the energy absorption values of tubes deforming in concertina mode were found to increase with foam filling. Babbage and Mallick [19] investigated the static axial crushing behavior of empty and epoxy foam-filled Al/filamentwound E-glass fiber reinforced epoxy composite tubes.

Despite the many experimental investigations conducted to understand the crushing behavior of foam-filled single metal tubes and empty composite tubes, the crushing behavior of constrained foam-filled multi-tube designs and metal/composite hybrid tubes has not been investigated as much. The present study was therefore conducted in order to determine the energy absorbing mechanisms of foam-filled multi-tube designs and metal/composite hybrid tubes. For this purpose two multi-tube packing geometries, hexagonal and square, and E-glass fiber woven fabric/polyester composite and Al metal tubes were chosen. Aluminum closed-cell foams of varying densities were prepared and used for the filling of thin-walled Al circular tubes and hybrid tubes. 


\section{Materials and Testing Methods}

Al closed-cell foam was prepared using the foaming from powder compacts (precursors) process. Foam plates of $8 \times 8 \mathrm{~cm}$ in cross-section and $3-4 \mathrm{~cm}$ in thickness and having densities ranging between 0.25 and $0.6 \mathrm{~g} \mathrm{~cm}^{-3}$ were prepared. Cylindrical foam samples were then core-drilled normal to the thickness of the plates. The detailed information on the foaming process and foam sample preparation is given in [20]. The density of Al foam filler was determined by simply dividing its weight to its volume. Deep-drawn thin $\mathrm{Al}$ tubes $(99.7 \% \mathrm{Al})$ used to construct hybrid tubes were produced by METALUM Company of Turkey and received in $25 \mathrm{~mm}$ diameter with $0.29 \mathrm{~mm}$ wall thickness. The yield and ultimate strength of the tube material were previously determined at a quasi-static strain rate of $\sim 1 \times 10^{-3} \mathrm{~s}^{-1}$ [27]. Tubes were machined to $27 \mathrm{~mm}$ in length using a diamond saw. Before the insertion of the Al tubes and foam fillers inside the composite tubes, the surfaces of the Al tubes and foam fillers were cleaned with acetone. E-glass fiber woven fabric/polyester composite tubes were prepared in house by handrolling of the pre-wetted woven-fabrics onto a surface-polished $25 \mathrm{~mm}$ steel bar. The reinforcing E-glass woven fabric, comprising $2 \times 2$ twill $\left(0^{\circ} / 90^{\circ}\right)$ fiber construction, had an areal density of $165 \mathrm{~g} \mathrm{~m}^{-2}$. The polymer matrix was prepared by mixing iso-polyester resin (blend of isophatalic acid and maleic anhydride or fumaric acid), with $1.5 \mathrm{wt} \%$ hardener solution containing $5 \mathrm{wt} \%$ Mek-peroxide and $0.28 \mathrm{wt} \%$ curing agent solution containing $6 \mathrm{wt} \%$ cobalt octoat. Initially, the fabric was cut into rectangular sections and then impregnated by the resin. Following the initial curing stage at room temperature, the composite tube was post-cured in a furnace at $120^{\circ} \mathrm{C}$ for $2 \mathrm{~h}$. After post curing, the tube was de-molded and finally sectioned into $27 \mathrm{~mm}$ long compression test samples using a slow-speed diamond saw. Empty tubes with fiber orientations $\pm 45^{\circ}$ in the tube long axis were prepared with wall thickness ranging between 0.56 and $0.65 \mathrm{~mm}$. The composite tubes were filled with Al closedcell foams of varying densities, ranging between 0.25 and $0.61 \mathrm{~g} \mathrm{~cm}^{-3}$. The foam density in foam-filled hybrid tubes was $0.4 \mathrm{~g}$ $\mathrm{cm}^{-3}$. Since the diameter of the foam fillers was the same as the inner diameter of the tubes, the foam fillers fitted tightly inside the composite and hybrid tubes without imposing any external pressure. A thin layer of polyester resin was used as the bonding agent between the filler and tubes surfaces. The weights and dimensions of tubes and fillers were measured before and after the filling. The weight of the adhesive used was calculated and found to vary between 3 and $6 \%$ of the total weight of the filled tubes.

Totally 12 groups of tests were performed. There were empty and foam-filled single Al and composite tubes (4), empty and foam-filled multi-tube-packed designs (4) and empty and foam-filled hybrid metal/composite tube (4). Figures 1(a-d) show sequentially empty composite and hybrid tubes and foam filled composite and hybrid tubes. Figures 2 (a-d) show sequentially empty hexagonal packed (MHE) and square packed (MSE) and foam-filled hexagonal packed (MHF) and square packed (MSF) multi-tube designs. Hexagonal packing consists of seven (Figure 1(a)) while square packing consists of four tubes (Figure 1(b)). Specially machined cylindrical and rectangular upper compression test platens fitted closely inside a circular Al die (75 mm in inner diameter, $2.5 \mathrm{~mm}$ in wall thickness and $35 \mathrm{~mm}$ in length) and a rectangular steel die (50x50 mm in crosssection) were used in the compression testing of the hexagonal- and square-packed empty and filled multi-tubes, respectively. Three hexagonal-packed foam-filled multi-tube designs were tested; (1) foam densities ranging between 0.4 and $0.47 \mathrm{~g} \mathrm{~cm}^{-3}$ with an average foam density of $0.44 \mathrm{~g} \mathrm{~cm}^{-3}$, (2) foam densities ranging between 0.34 and $0.4 \mathrm{~g} \mathrm{~cm}^{-3}$ with an average foam density of $0.38 \mathrm{~g} \mathrm{~cm}^{-3}$ ) and (3) foam densities ranging between 0.51 and $0.6 \mathrm{~g} \mathrm{~cm}^{-3}$ with an average foam density of $0.55 \mathrm{~g}$ $\mathrm{cm}^{-3}$ ). While two square-packed foam-filled multi-tubes with average foam densities of 0.31 and $0.28 \mathrm{~g} \mathrm{~cm}^{-3}$ were tested. The foam densities in square-packed multi-tube designs ranged between 0.3 and $0.33 \mathrm{~g} \mathrm{~cm}^{-3}$ for the first group and 0.26 and 0.29 $\mathrm{g} \mathrm{cm}^{-3}$ for the second group of tubes.

The length of the tubes in all configurations was $27 \mathrm{~mm}$, determined by the thicknesses of Al foam plates prepared. Quasistatic compression tests on empty and foam-filled tubes and foam samples were conducted in a displacement controlled SHIMADZU AG-I universal testing machine with a displacement rate of $2 \mathrm{~mm} \mathrm{~s}^{-1}$, using an eccentric compression upper test platen. Test platens were lubricated in order to reduce the radial frictional forces. The average crushing load $\left(P_{a}\right)$ and $S A E$ values were calculated using following relations:

$$
\mathrm{P}_{\mathrm{a}}=\frac{\int \mathrm{P} \mathrm{d} \delta}{\delta}
$$

and

$$
\mathrm{SAE}=\frac{\int \mathrm{P} d \delta}{\mathrm{m}}
$$

where, $\mathrm{P}, \delta$ and $\mathrm{m}$ are the load, the displacement and the mass of the tube(s), respectively. 

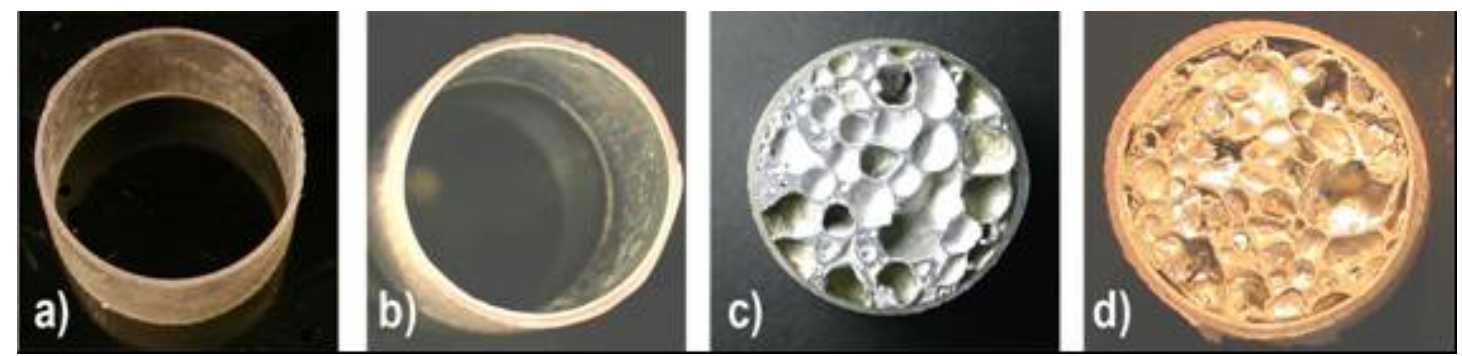

Figure 1. (a) empty composite, (b) empty hybrid, (c) foam-filled composite and (d) foam-filled hybrid tubes
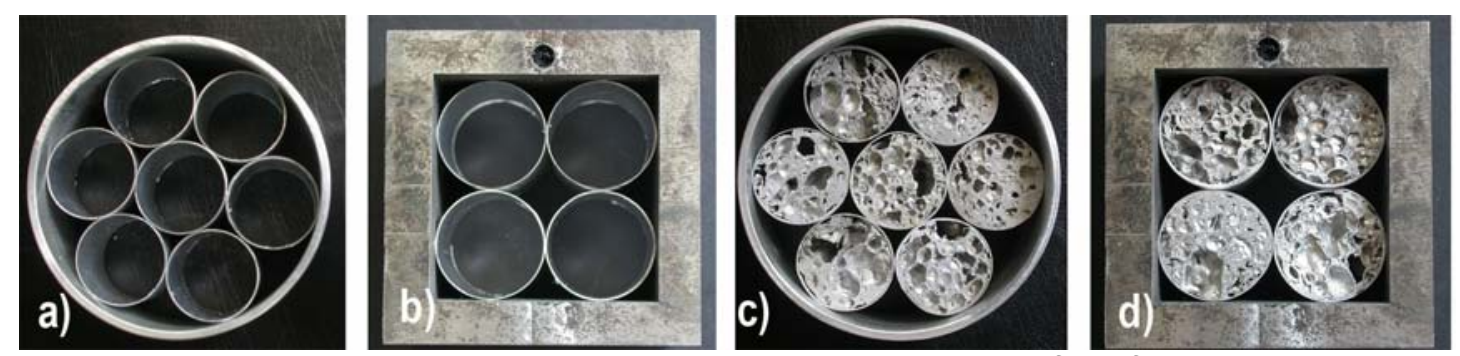

Figure 2. Multi tube designs; empty (a) hexagonal-packed and (b) square-packed and foam-filled (c) hexagonal-packed and (d) square-packed multi tube designs

\section{Results}

The load-displacement curve of $0.27 \mathrm{~g} \mathrm{~cm}^{-3} \mathrm{Al}$ foam-filled single tube is shown in Figure 3 together with the load-displacement curves of empty tube+foam (the sum of the loads of empty tube and foam), empty tube and Al foam. In the same figure the average crushing loads of filled tube, empty tube+foam and empty tube are also shown by the dotted lines. The average crushing loads of Al foam-filled tube were calculated between the displacements of 2 and $10 \mathrm{~mm}$ (corresponding $7 \%$ and $37 \%$ deformation) since at increasing displacements Al foam filler crushing load increased sharply above the foam plateau load. The interaction effect of foam filling is clearly seen in Figure 3 as the crushing and average crushing loads of the filled tube are higher than that of empty tube+foam. The interaction effects were also found in 0.35 and $0.43 \mathrm{~g} \mathrm{~cm}^{-3}$ foam-filled tubes. Regardless the Al foam density used, the foam filling shifted the deformation mode of empty tube from diamond (Figure 4(a)) into concertina (Figure 4(b)). The shift in the deformation mode was attributed to the tube wall thickening effect of the filler. It is also noted that the folding always started at one of the ends of the tube and progressively proceeded along the tube length through the other tube end. The foam filling also increased the number of folds formed in foam filled tubes from 4 (empty tube) to 6 and decreased the fold length.

The tubes of hexagonal and square packed empty multi-tube designs deformed in diamond mode of deformation similar to the single empty tubes (Figures 5(a) and (b)). The tubes of multi tube designs seen in Figures 5(a) and (b) however show more irregular patters of diamond mode of deformation as compared with single empty tubes. The irregular deformation mode in empty multi-tube designs is attributed to the constraint effects of the adjacent tubes and the constraint outer tube walls. In foam-filled multiple tubes, the deformation mode switched from diamond into concertina mode, the same with that of foamfilled single tubes (Figures 5 (c) and (d)). The constraint effect of outer tube walls is clearly seen in both designs; tubes touching to the outer tube walls became slightly elliptical in hexagonal packing while in square packing the tubes became slightly rectangular in cross-sections as seen in Figures 5(c) and (d), respectively.

Typical load-displacement curves of hexagonal packed empty and foam-filled multiple tubes are shown sequentially in Figures $6(a)$ and (b). As is seen in Figure 6(a) the crushing and average crushing loads of empty multi-tube designs are higher than those of the sum of the crushing and average crushing loads of the equal number of single empty tubes (crushing and average crushing loads of single empty tube are multiplied by the numbers of tubes in multi-tube designs). It was found that the differences between the average crushing loads of empty single tubes determined at different percent deformations were less than $5 \%$. The increase in the average crushing loads of multi tube designs of the empty tubes were calculated at $50 \%$ deformation and found 0.87 and $0.4 \mathrm{kN}$ on the average for MHE and MSE designs respectively. These corresponded to $12 \%$ and $10 \%$ increase in the crushing loads of empty tubes. Similar to the filled single tubes, the crushing load values of foamfilled multi-tube designs were higher than those of empty tubes+foams as depicted in Figure 6(b) for hexagonal packing. In the foam-filled multi-tube designs, the contributions to the crushing loads may include (a) empty tube crushing loads (b) the foam filler loads (c) the strengthening coefficient of the foam filled single tubes and lastly (d) the frictional loads and constraint effects imposed by the tubes and constraint outer tube walls. 


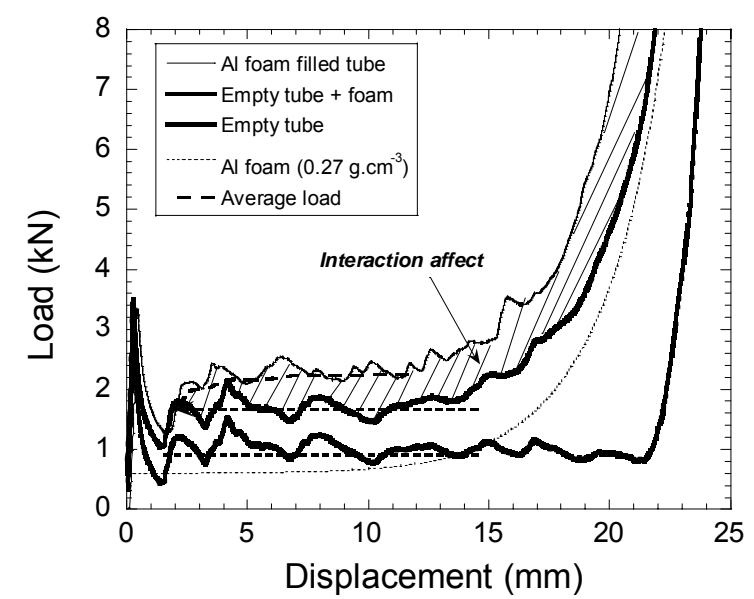

Figure 3. Load and average crushing load-displacement curves of $0.27 \mathrm{~g} \mathrm{~cm}^{-3}$ foam-filled single Al tube, empty tube, empty tube+foam and foam
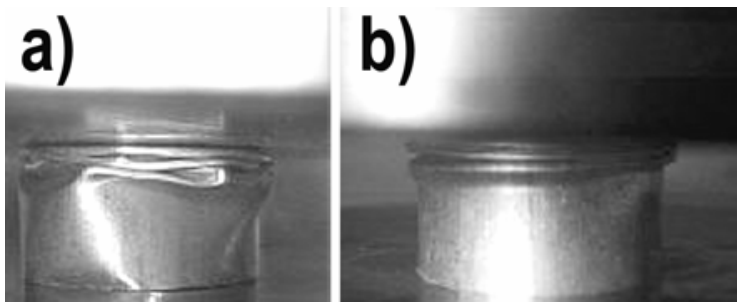

Figure 4. Images of (a) diamond mode of deformation in empty Al tube and (b) concertina mode of deformation in Al foamfilled $\left(0.35 \mathrm{~g} \mathrm{~cm}^{-3}\right)$ tube at $50 \%$ percent deformation

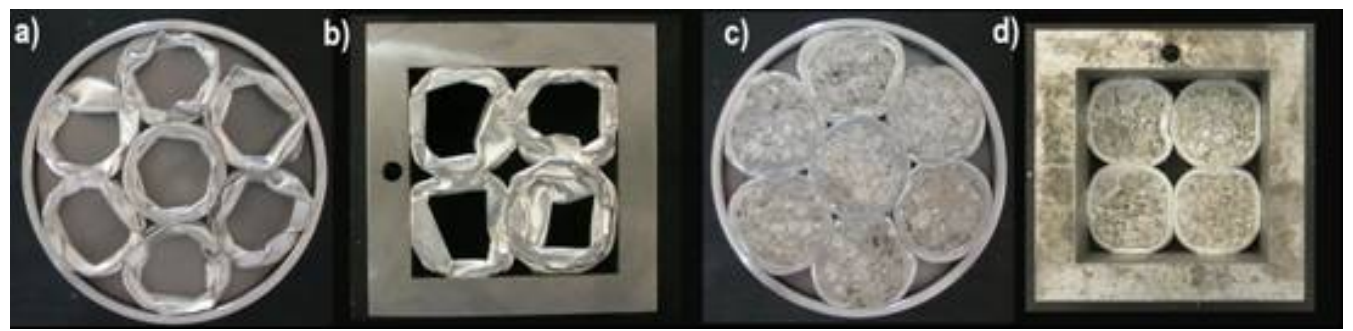

Figure 5. Crushed empty multi-tube designs; empty (a) hexagonal and (b) square packed and foam-filled (c) hexagonal and (d) square packed

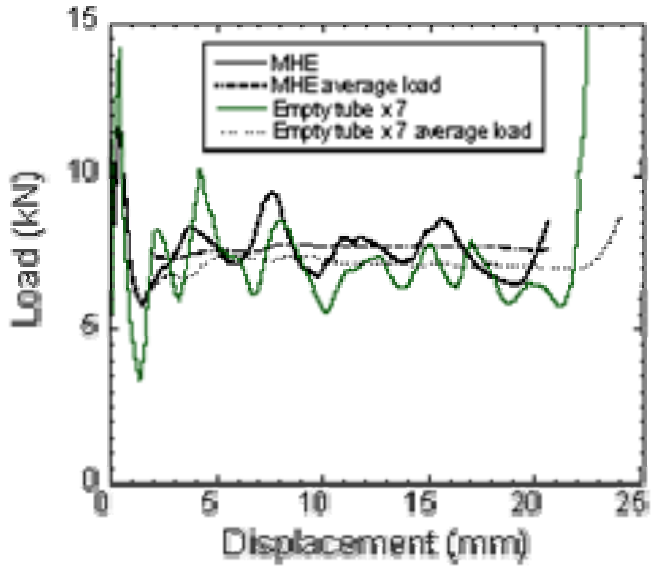

(a)

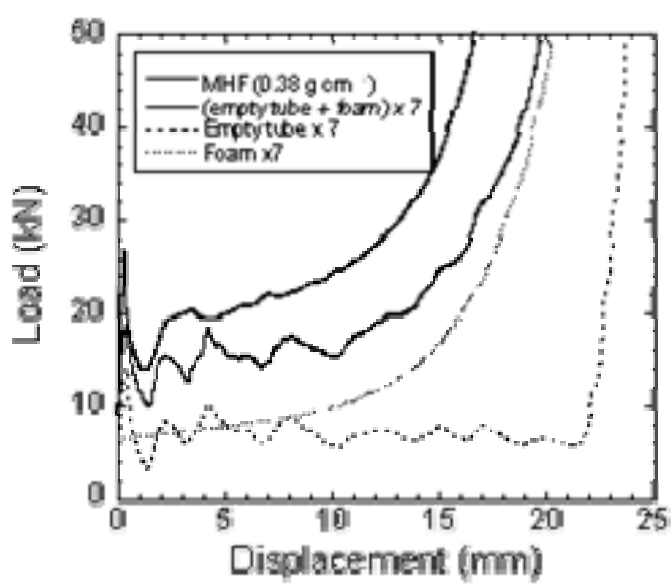

(b)

Figure 6. Load-displacement curves of (a) empty (MHE) and (b) Al foam-filled hexagonal (MHF) packed multi-tube designs 
The strengthening coefficients of foam filling can be expressed for foam-filled single tubes as;

$$
C=\frac{P_{s f}-P_{s e}}{P_{f}}
$$

and for foam-filled multi tubes as

$$
C=\frac{P_{m f}-P_{m e}}{P_{f}}
$$

where $P_{s f}$ and $P_{s e}$ are the average crushing loads of foam-filled and empty single tubes, $P_{m f}$ and $P_{m e}$ are the average crushing loads of foam-filled and empty multiple tubes and $P_{f}$ is the foam filler plateau load, respectively. The strengthening coefficient of foam-filled single tubes was predicted. The increase in the average crushing load in foam-filled single tubes $\left(\Delta P=P_{\text {sf }}-P_{\text {se }}\right.$, where $P_{\text {se }}$ is $0.99 \mathrm{kN}$ ) was drawn as function of foam plateau load. The average crushing load of foam-filled single and multiple tubes and plateau load of the foam fillers increase as the deformation increases; therefore, the average crushing loads of the filled single and empty tubes and plateau load of foam fillers were determined at a constant displacement corresponding to $20 \%$ of deformation. The strengthening coefficient of foam-filled single tubes is predicted to be 1.65 . This value of strengthening coefficient is very similar to the previously determined strengthening coefficient value for square Al tubes (1.8) [5]. In the calculations of the strengthening coefficient of the multi-tube designs the foam plateau load was taken as the plateau load of the average foam density, which was predicted through an interpolation route. The tested foams plateau loads ( $20 \%$ deformation) was drawn as function of the foam density (between 0.25 and $0.6 \mathrm{~g} \mathrm{~cm}^{-3}$ ), then the plateau load were fitted with the foam density thorough a power-law relation. The predicted strengthening coefficients of multi-tube designs range between 1.85 and 2.64. The increase in foam filler density also tends to increase the strengthening coefficients of the multitube designs.

In the tested empty composite tubes two crushing modes were clearly distinguishable: (a) progressive crushing through external spraying and (b) catastrophic failure via compressive shear and/or axial splitting of the tube wall. Figures $7(\mathrm{a}-\mathrm{c})$ show sequentially progressive crushing of the empty composite and hybrid tubes and catastrophic failure in foam filled hybrid tube. These crushing modes are in accord with the crushing modes proposed previously by Hamada and Ramakrishna [9]. Among five tests only one of the empty composite tube samples failed by catastrophic failure. As shown in Figure 8 , the empty composite tube deforms elastically until the peak-load, thereafter localized deformation triggers near one of the ends of the tube and progresses through the length of the tube. Following the initial peak-load due to the localization of the deformation in an area near one of the tube ends the deformation switches to progressive crushing mode and the load values increase to an average value (average crushing load) as shown in Figure 8. Further deformation of the tube proceeds with outward splaying mode. Similar deformation modes were also observed previously in a glass fiber/epoxy composite [12]. The mean average crushing loads of the tubes were calculated in the progressive crushing mode of the load-displacement curves, after point 4 of Figure 8 .

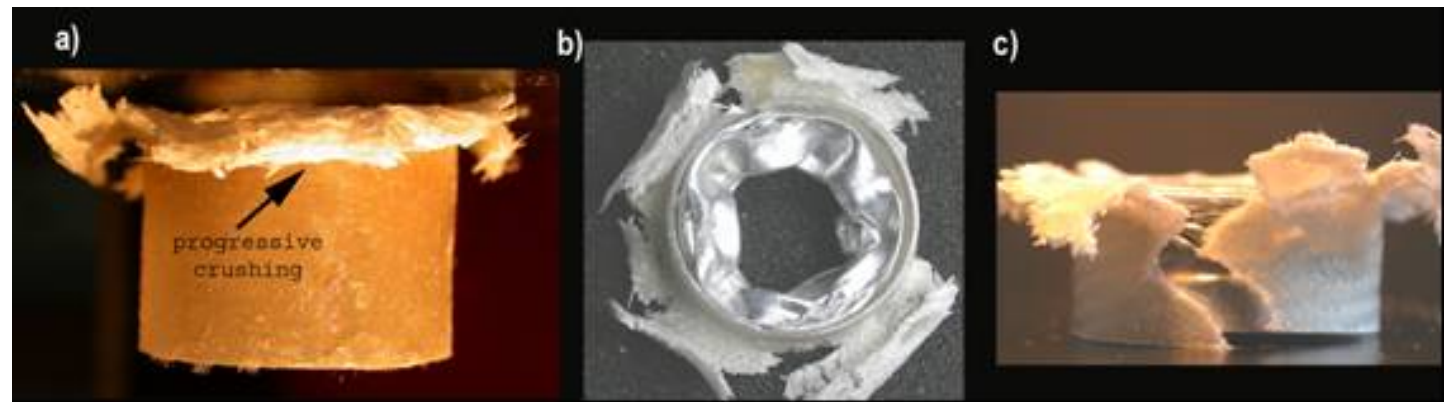

Figure 7. Progressive crushing mode of (a) empty composite and (b) empty hybrid tube and (c) catastrophic failure of the foam filled hybrid tube 


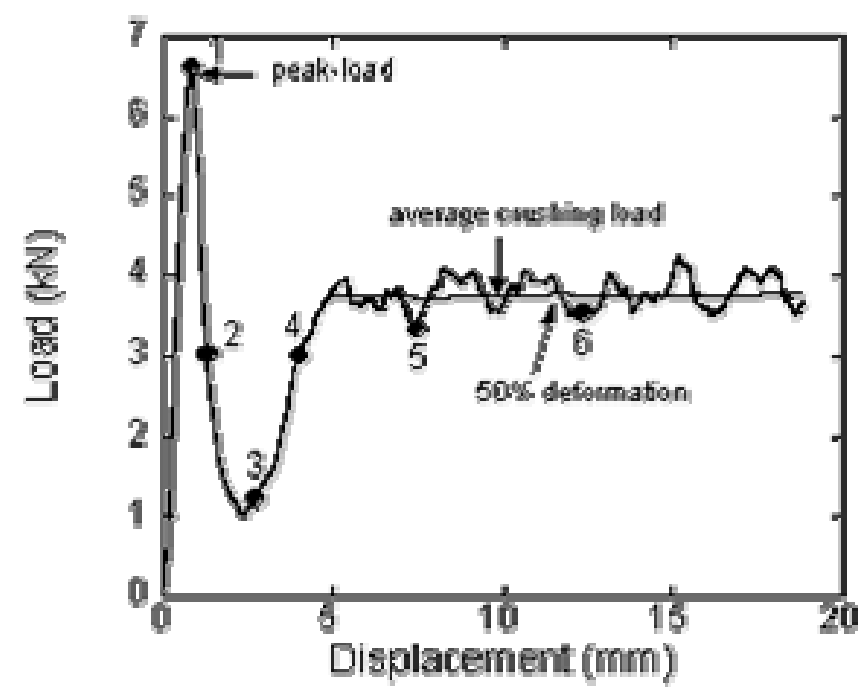

Figure 8. Load-displacement curve of an empty composite tube of $0.55 \mathrm{~mm}$ thick showing progressive crushing mode

Total 8 hybrid tubes were tested and similar to empty composite tubes, two crushing modes were observed; progressive (6 samples) and compression shear (2 samples). In progressive crushing mode of hybrid tubes are very much similar to the crushing behavior of empty composite tube in the initial deformation region. In hybrid tubes it is also noted that metal tube folding triggers at the same place that composite tube deformation triggers for the progressive crushing. Similar to empty composite tube crushing behavior, the deformation mode switches to progressive crushing mode and load values reach a constant average crushing load following to the initial deformation region. Further deformation proceeds with outward splaying mode similar to empty composite tubes. The peak-loads and crushing loads of the hybrid tube samples failed by compression shear are relatively lower than those of the samples failed by progressive crushing mode. The peak-loads in catastrophically failed samples were 7.4 and $6.4 \mathrm{kN}$, while in progressively failed samples ranged between 9.1 and $11.2 \mathrm{kN}$. The mean average crushing loads of hybrid tubes decreased from $6.2 \mathrm{kN}(5.17-7.2 \mathrm{kN})$ in progressively failed samples to 3.15 and $3.2 \mathrm{kN}$ in catastrophically failed samples.

Since the crushing modes of composite tube of the hybrid and empty composite tubes are very much similar, it may be concluded that the crushing behavior of hybrid tubes is dominated in a great extent by the composite tube crushing behavior. The deformation mode of Al tube is however changed significantly in hybrid tubes. Figure 7(b) shows the deformed shape of a hybrid tube in progressive crushing mode at $20 \mathrm{~mm}$ displacement, taken from the back of the crushing direction. Although the deformation mode of Al tube in hybrid tubes is similar to the diamond mode of empty tube, the shape and the geometry of the folds are not as homogeneous as the empty tube diamond mode. The change in the deformation mode of Al tube in hybrid tube is due to the prevention of the outer folding of Al tube. This is clearly seen in Figure 7(b), where Al tube folds are entirely through the inside. Although the empty tube shows an eight-cornered diamond mode of deformation, the number of corners increases in the hybrid tubes. Since the composite tube wall in the samples failed by compression shear was partly effective in constraining $\mathrm{Al}$ tube, the diamond mode deformation in these samples was much more similar to that of empty Al tube except again the numbers of the folds increased in the hybrid tube particularly at the later stages of the deformation.

In foam-filled hybrid tubes, the folding of metal tube is constrained both by the foam filler and the outer composite tube. The resistance imposed by the filler and the composite was however found to be effective only in the initial stages of the deformation, since at the later stages of deformation the composite failed by axial cracks as shown in Figure 7(c). The crushing behavior of the composite tube shows in fact a mixture of progressive and catastrophic failure modes. In empty hybrid tubes, although metal tube crushes in inhomogeneous diamond mode, it deforms in concertina mode in the foam-filled hybrid tube. The concertina folds in a foam-filled Al tube were previously shown to be uniform in shape and thickness and mostly outward of the filler [2]. While in hybrid tubes the folds are not as regular as in the case of foam-filled Al tube and tend to form through the inside of the foam. The irregular folds are most probably resulted from the entrance of the fractured composite pieces in between the folds. This also confirms that although composite tube fails by axial cracks it remains attached to Al tube and provides a partial confinement to Al tube folding.

The compression load-displacement curves of foam-filled composite tubes of increasing foam densities are shown in Figure 9(a), together with the load-displacement curve of an empty composite tube. As the foam density increases, as clearly seen in this figure, peak-load and crushing load values increase. It is also noted in Figure 9(a) that foam filling: (a) induces a continuously increasing load values following the peak-loads and (b) reduces the magnitude of load drop following the peakload. The latter suggests that foam filling induces stable tube crushing triggering and progression. This was also supported by 
the observed lack of the compressive shear type failure in the tested ten foam-filled composite tubes within the studied foam density range. The crushing behavior of the foam filled composite tubes is very similar to that of the progressively crushed empty tubes except the magnitude of the load drop following the peak-load decreases in the filled tube as noted previously. Although foam filling increases the crushing loads in the initial deformation region, it is ineffective in increasing the crushing loads of the composite tube in the progressive crushing region over those of foam + empty composite tube as shown in Fig. 9 (b) for a $0.35 \mathrm{~g} \mathrm{~cm}^{-3}$ foam-filled composite tube. Once the tube crushing triggers from one of the ends of the tube, the bonding between foam and tube presumably separates or partially separates; therefore, the deformation of the tube and foam proceeds separately, leading to no interaction between tube and foam.

Figure 10(a) shows the load-displacement curves of a hybrid tube deforming in progressive crushing mode, empty composite and $\mathrm{Al}$ tubes and the mathematical sum of the loads of the empty composite and empty Al tube. The higher load values of hybrid tube than those of empty composite tube + empty Al tube shown in this figure basically prove the interaction between Al and composite tube in hybrid tubes. The interaction, as stated previously, is a result of the constraint of the composite to the outward folding of Al tube. The load-displacement curves of foam-filled $\left(0.4 \mathrm{~g} \mathrm{~cm}^{-3}\right)$ hybrid tubes are shown in Figure 10 (b) together with load-displacement curves of empty composite and empty hybrid tubes. As shown in this figure foam-filled hybrid tubes show similar load values with those of empty hybrid tube, proving that the foam filling is not advantageous over the empty hybrid tubes. However, further experimentation on the thicker and lower density of foam filler may reveal specific tube and filler combinations, inducing progressive crushing mode of the composite tube.

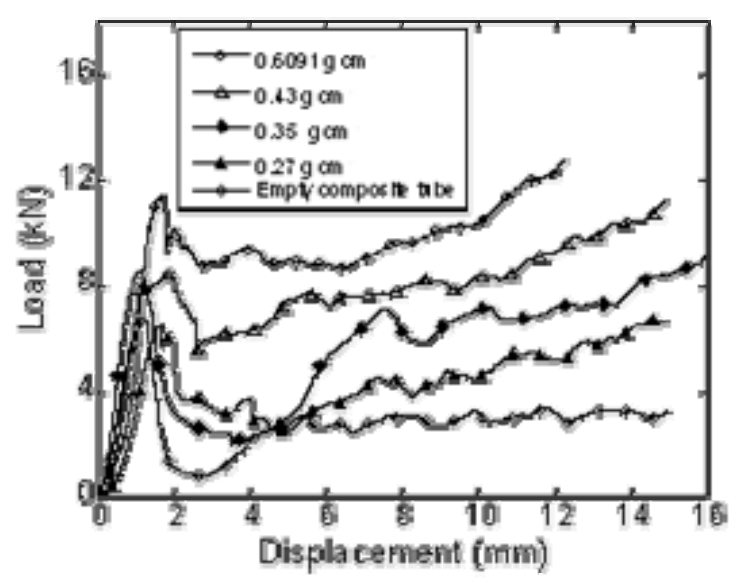

(a)

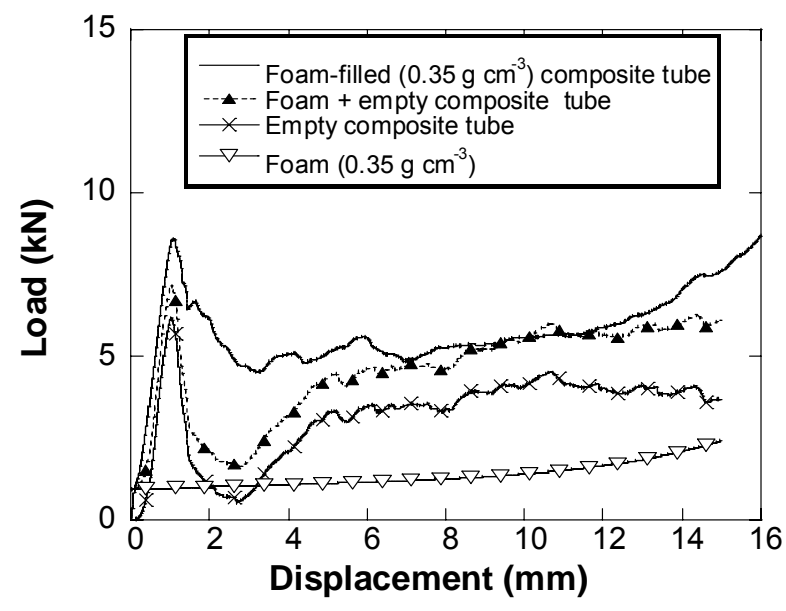

(b)

Figure 9. Load-displacement curves of (a) foam-filled and empty composite tubes and (b) comparison of foam-filled composite tube crush loads with empty composite tube and Al foam and the sum of the loads of foam and empty composite tube

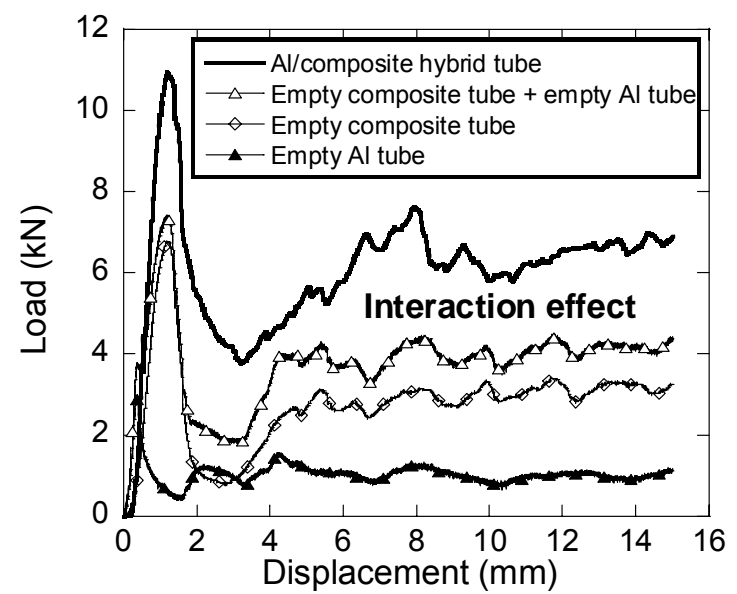

(a)

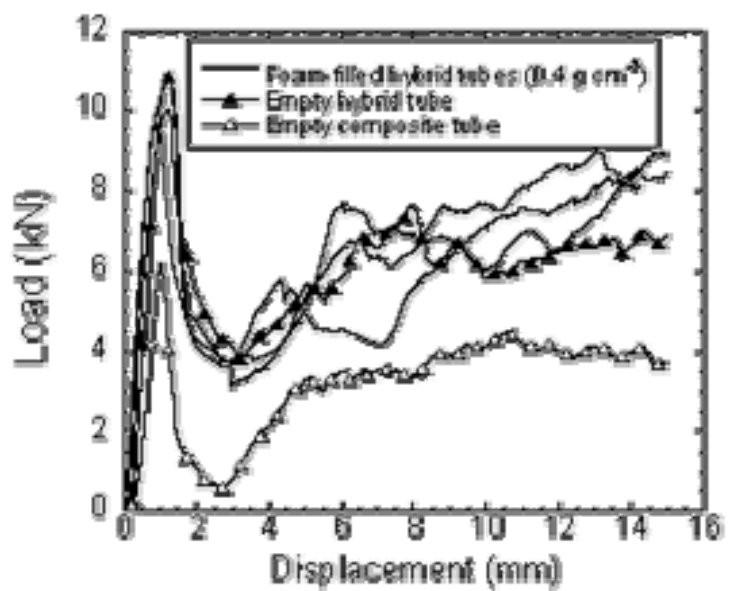

(b)

Figure 10. Comparison of crushing loads of hybrid tubes with empty composite tube and empty Al tube and empty composite tube + empty Al tube and (b) Load-displacement curves of foam-filled hybrid, empty hybrid and composite tubes 


\section{Analysis of the Results}

The relative increase in the average crushing loads of empty multi-tube designs is simply a result of (a) the constraining effects of the adjacent tubes and the constraint outer tube walls and (b) the frictional forces between the tube walls and tubes and outer tube wall. These effects are however greater in MHE design due to a higher number of packed tubes. In multi-tube designs, besides the resistance of the filler to tube wall folding, the tube and the constraint tube walls provide additional resistance to the deforming tubes walls. At the tube wall-constraint tube wall contact regions, the tube wall folding occurred completely inward, providing likely additional strengthening mechanism in multi-tube designs. The folding mechanisms in multitube designs are rather complex and the contributions of few different mechanisms to the average crushing load are however not known precisely. The number of tubes packed in multi-tube designs may also change the extent of these contributions. Further experimentation and microscopic studies accompanied with modeling will therefore be conducted in order to identify the deformation mechanism more clearly and to calculate the extent of contributions of each mechanism to the average crushing load.

Although energy absorption increases with foam filling in foam-filled single Al tubes, the SAE values of the filled single tubes are lower than that of empty tube (Figures $11(\mathrm{a})$ and (b)) until about the displacements of $15-20 \mathrm{~mm}$; thereafter, the foam filling becomes more efficient than empty tube. This effect, the increase in SAE values of filled tubes over the empty tube at increasing displacements, is simply a result of the increase of the foam filler density at increasing deformation ratios. It was previously shown that there is a critical total tube mass and the corresponding critical foam density above which the use of foam filling becomes more efficient than empty tube [1, 2]. The SAE values of empty multi-tube designs however exceed that of the single empty tube after $10 \mathrm{~mm}$ displacement as shown in Figures. 11(a) and (b). This is due to the increasing constraint effects and frictional forces between tubes and tubes and outer tube walls as the deformation increases. The foam filling of the multi-tube designs is however not effective in increasing SAE values over those of single empty tube and empty multi-tube designs (Figure 11 (a)). At similar foam filler densities multi-tube designs are energetically more effective than Al foam-filled single tubes for both hexagonal and cubic packed designs (Figure 11(b)). Note also in Figures 11(a) and (b) that as the deformation increases, the foam filling of multi-tube designs becomes energetically more efficient than empty tubes partly due to increasing foam density and partly due to increased constraint effects and frictional forces with increasing deformation.

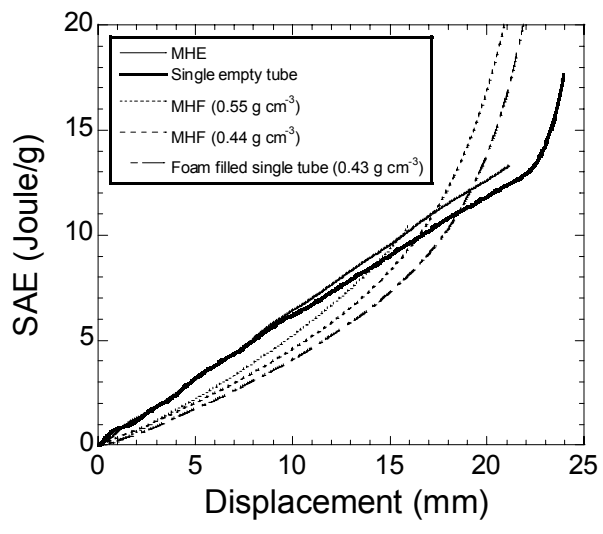

(a)

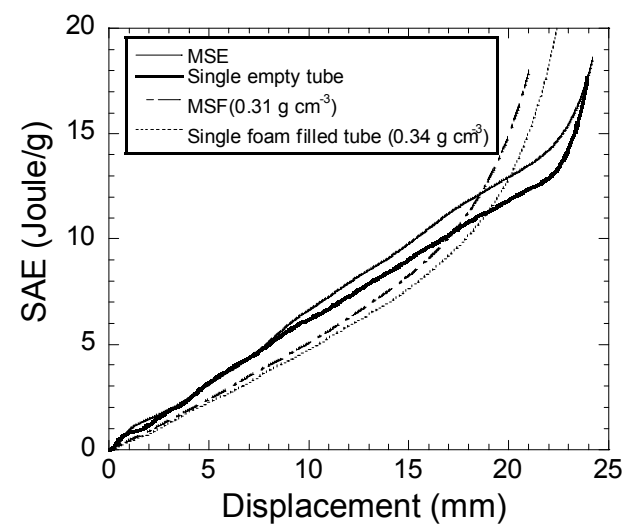

(b)

Figure 11. Comparison of SAE values between (a) empty tube and MHF designs and (b) empty tube and MSF designs

It is known that progressive crushing mode can be achieved by chamfering one of the ends of the composite tube [9, 21]. When the compressive load is applied, the load at the tip of the chamfer end of the tube increases significantly, leading to micro fracture initiating and the progression of the crushing zone. The observed progressive crushing mode triggering in the studied empty composite tubes is believed partly due to the imperfections at the tube end faces presumably introduced during the sectioning of the tubes with diamond saw. Delaminations introduced during the sectioning of the composite tubes are likely to trigger the deformation from one of the ends of the tube. The imperfect contact forces between the tube and compression test platen may also increase the load locally in the contact area, leading to the initiation and progression of the crushing zone from one of the ends of the tube. The comparison of typical SAE values of the empty hybrid tubes with those of empty composite tube, empty Al tube and empty composite tube + empty Al tube is shown in Figure 12(a). Since the energy absorption in the initial region of the deformation is predominantly affected by the triggering process only the load values of progressive crushing region were included in the calculations. The hybrid tube shows higher SAE values than those of empty composite tube + empty Al tube, showing, as in the case of load values, an interaction effect between composite tube and Al tube. A simple calculation based on the mean average loads of empty composite tube, empty Al tube and empty hybrid tube shows an increase in the mean average crushing load of hybrid tubes as high as 2.3 times of the mean average crushing load of empty Al tube. This value is also comparable with experimentally found strengthening coefficients of $\mathrm{Al}$ foam-filled $\mathrm{Al}$ tubes, ranging between 1.7 and 2.8; the higher value of strengthening arises from the use of adhesive between foam filler and tube 
[3]. In Figure 12(b), typical SAE values of foam-filled composite, foam + empty composite tube and foam are shown together with SAE values of empty composite tube. As shown in Figure 12(b), foam filling of composite tube gives similar SAE values with those of foam + empty composite tube, showing again no interaction between foam and tube. The foam filling of hybrid tubes results in SAE values similar to those of empty Al tubes, showing the ineffectiveness of foam filling in these tubes. However, foam-filled hybrid tubes show far less SAE values than empty composite and empty hybrid tubes.

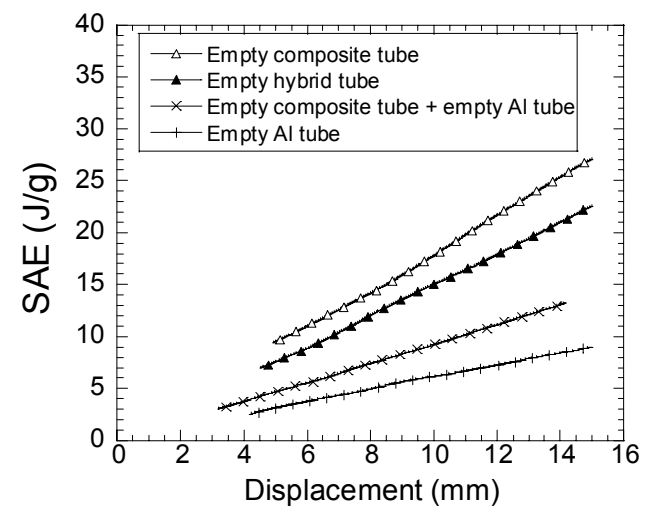

(a)

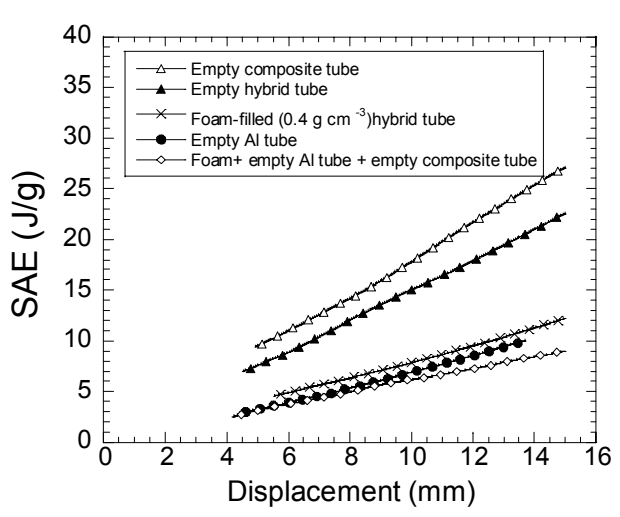

(b)

Figure 12. Comparison of SAE values of (a) empty hybrid tube with empty composite, empty Al and composite + Al tubes, (b) foam-filled composite tube with empty composite tube, foam and empty composite tube +foam and (c) foam-filled hybrid tube with empty composite, hybrid and Al tubes, foam and foam + empty Al tube + empty composite tube

\section{Conclusions}

An experimental study was conducted in order to determine the effect of foam filling on the quasi-static crushing behavior of multi metal tube and woven fabric composite and Al metal/composite hybrid tubes. The effects of foam filling in single Al tubes were (a) to increase the average crushing load over that of the tube (alone) + foam (alone) and (b) to decrease the fold length. Similar to single empty tubes, the foam filling shifted the deformation mode of empty tubes from diamond to concertina mode in multi-tube designs. The effect of multiple tube packing was seen as the increased crushing and average crushing load values over the sum of the average crushing loads of the equal number of single empty and foam-filled tubes. The increase in the average crushing loads of multi-tube designs over the single tubes was attributed to the constraint effects and frictional forces between tubes and tubes and outer tube walls. The foam density was further found to increase the interaction effects in multi-tube designs. Although foam filling in single and multi tubes resulted in higher energy absorption than the sum of the energy absorptions of the tube(s) and foam(s), it was not effective in increasing the SEA values over the empty single tube and empty multi-tube designs. At similar foam filler densities multi-tube designs were however energetically more effective than Al foam-filled single tubes for both hexagonal and cubic packed designs.

Two crushing modes, progressive and catastrophic, were observed in the testing of empty composite and empty hybrid tubes. The progressive crushing mode resulted in higher crushing loads, hence higher SAEs. In empty hybrid tubes, the deformation mode of Al tube was found to be a more complex form of the diamond mode of deformation, leading to higher SAE values than the sum of the SAEs of empty composite tube and empty metal tube. The increased load and SAE values of hybrid tubes were attributed to the interaction between composite tube and Al tube. The foam filling of the composite tubes was found to be ineffective in increasing foam-filled tube crushing loads over the sum of the crushing loads of empty composite and foam in the progressive crushing region. However, the foam filling induced a more stable tube crushing trigger and progression. In the foam-filled hybrid tubes, the composite tube failed by axial splitting due the resistance imposed by the foam filler to the metal tube inward folding; therefore, foam filling was ineffective in increasing crushing loads; hence, SAE values over those of empty composite tube + empty Al tube + foam.

\section{Acknowledgments}

The authors would like to thank the Scientific and Technical Council of Turkey (TUBITAK) for the grant \#MISAG-227 and METALUM Company of Turkey for supplying Al tubes.

\section{References}

1. Santosa S. and Wierzbicki T. "Crash behavior of box columns filled with aluminum honeycomb or foam." Computers and Structures 68, 343-367(1998). 
2. Kavi H., Toksoy AK. And Guden M. "Predicting energy absorption in a foam-filled thin-walled aluminum tube based on experimentally determined strengthening coefficient." Materials \& Design 27, 263-269(2006).

3. Toksoy A.K., Tanoglu M., Guden M. and Hall I.W. "The effect of adhesive on the strengthening of aluminum foam-filled circular tubes." Journal of Materials Science, 39,1503-1506(2004).

4. Seitzberger M., Rammerstorfer F.G., Gradinger R., Degischer H.P., Blaimschein M. and Walch C. "Experimental studies on the quasi-static axial crushing of steel columns filled with aluminum foam." International Journal of Solids and Structures , 37,4125-4147(2000).

5. Santosa S.P., Wierzbicki T., Hanssen A.G. and Langseth M. "Experimental and numerical studies of foam-filled sections." International Journal of Impact Engineering, 24, 509-534(2000).

6. Hanssen A.G., Langseth M. and Hopperstad O.S. "Static and dynamic crushing of square aluminium extrusions with aluminum foam filler." International Journal of Impact Engineering, 24, 347-383(2000).

7. Guillow S.R., Lu G. and Grzebieta R.H. "Quasi-static axial compression of thin-walled circular aluminum tubes." International Journal of Mechanical Sciences, 43, 2103-2123(2001).

8. Singace A.A. "Collapse behaviour of plastic tubes filled with wood sawdust." Thin-Walled Structures,37, 163-187(2000).

9. Ramakrishna S. "Microstructural design of composite materials for crashworthy structural applications." Materials \& Design, 18,167-173(1997).

10. Harte A.M.and Fleck N.A. "Deformation and failure mechanisms of braided composite tubes in compression and torsion." Acta Materialia, 48, 1259-1271(2000).

11. Hamada H. and Ramakrishna S. "Energy absorption characteristics of crash worthy structural composite materials." Engineering Materials, 141-143,585-620(1998).

12. Saito H., Inai R., Yokoyama A.and Hamada H. "Basic study of progressive crushing mechanism. "Engineering Materials 177-180:321-326(2000).

13. Chiu C.H., Tsai K.and Huang W.J. "Effects of braiding parameters of energy absorption capability of triaxially braided tubes." Journal of Composite Materials, 32,1964-1983(1998).

14. Turner T.A., Warrior N.A, Robitaille F. and Rudd C.D. "The influence of processing variables on the energy absorption of composite tubes." Composites Part A: applied science and manufacturing, 36,1291-1299(2005).

15. Warrior N.A., Turner T.A., Robitaille F. and Rudd C.D. "The effect of interlaminar toughening strategies on the energy absorption of composite tubes. " Composites Part A: applied science and manufacturing, 35,431-437(2004).

16. Warrior N.A., Turner T.A., Robitaille F. and Rudd C.D. "Effect of resin properties and processing parameters on crash energy absorbing composite structures made by RTM." Composites Part A: applied science and manufacturing, 34, 543550(2003).

17. Zeng T., Fang D.and Lu T. "Dynamic crashing and impact energy absorption of 3D braided composite tubes." Materials Letters, 59,1491-1496(2005).

18. Shin K.C., Lee J.J., Kim K.H., Song M.C.and Huh J.S. "Axial crush and bending collapse of an aluminum/GFRP hybrid square tube and its energy absorption capability." Composite Structures, 57,279-287(2002).

19. Harte A.M., Fleck N.A. and Ashby M.F. "Energy absorption of foam-filled circular tubes with braided composite walls." Eur. J. Mech.A/Solids, 19,31-50(2000).

20. Guden M. and Yuksel S. "Compression behavior of SiC-particulate foams produced by foaming from powder compacts. Journal of Materials Science, 41,4075-4084(2006).

21. Jimenez M.A., Miravete A., Larrode E. and Revuelta D. "Effect of trigger geometry on energy absorption in composite profiles." Composite Structures, 48,107-111(2000). 